\title{
Trilateral Location based Maximum Weighted Directive Spanning Tree for Optimal Routing in loV
}

\author{
S. SUGUNA DEVI, A. BHUVANESWARI \\ Department of Computer Science, Cauvery College for Women, Trichy-18 \\ Affilated to Bharathidaasan University, India, (e-mail: s.sugunadevi.it@cauverycollege.ac.in, bhuvaneswari.it@cauverycollege.ac.in) \\ Corresponding author: S. Suguna Devi (e-mail: s.sugunadevi.it@ cauverycollege.ac.in).
}

\begin{abstract}
: ABSTRACT Internet of Vehicles (IoV) is one of the developing models in the Vehicular adhoc networks (VANETs) with the vast improvement of communication technologies. In order to improve data transmission among the multiple communities without link breakage, a novel Trilateral Location Identified Maximum Weighted Directive Spanning Tree (TLIMWDST) technique is introduced. The proposed TLIMWDST technique consists of two major phases, namely location identification and optimal path identification to improve the reliability of data transmission from source vehicle to destination vehicle. In the first phase, the location of the neighboring vehicles is identified by applying a trilateration technique. After the location identification, an optimal route path between the source and destination is identified using Maximum Weighted Directive Spanning Tree (MWDST) through the intermediate nodes. The performance of the TLIMWDST technique is assessed through simulation as compared to the previous path selection techniques in terms of different routing metrics such as packet delivery ratio, packet loss rate, end-to-end delay and throughput with respect to the number of data packets.
\end{abstract}

: KEYWORDS IoV; trilateration multiple communities; neighboring vehicles; location Identification Maximum Weighted Directive Spanning Tree; Optimal Path discovery.

\section{INTRODUCTION}

$\mathrm{T}$ HE enormous growth of vehicles on roads, IoV has become one of the significant field providing better explanations for the smart transportation system. At present, several methods are focusing mainly on the construction and improvement of IoV due to its significance in an Intelligent Transportation System (ITS). Precisely, routing protocols are becoming the most significant issue in IoV application for improving the stable path to gain better performance between vehicles interaction. An efficient routing algorithm is required for reliable path selection in the rapid topology changes and frequent link disconnections due to the high mobility of vehicles.

The community aware mechanism on the Internet of vehicles (CIoVS) was developed [1] to distribute the data packets based on vehicles for long-distance communication between the vehicles. Though the designed CIoVS minimizes the delay time, the higher packet delivery ratio was not achieved. A Reliable Path Selection and Packet Forwarding Routing Protocol (RPSPF) was designed in [2] to discover an optimal route for transmitting the packets towards their particular destinations by taking into consideration the link connectivity and the shortest optimal route path. But, the performance analysis of the packet loss rate was not estimated.

An Identical Destination Based Community on the Internet of Vehicles (IDCIoV) was proposed in [3] to discover the optimal path. The method does not provide minimal end to end delay for data communication. Heterogeneous network structural design was introduced in [4] to enhance reliable communication and support seamless data transmission. The developed architectural design minimizes the delay but the performance of reliable data delivery was not evaluated. 
A reliable emergency message distribution protocol was introduced in [5] to minimize the extra delays due to the exchange of control messages. The protocol uses different handshake procedures for road segments to preserve the low signaling overhead. Besides, it reduces the one-hop delay during the data transmission but the packet loss was not minimized. A Hybrid Emergency Message Transmission (HEMT) scheme was introduced in [6] to support the flexibility of reliable and fast transmission emergency messages through the SDN-facilitated central controller and RSU unit. The designed HEMT scheme failed to reduce the delay at a required level.

A position-based data dissemination approach was developed in [7] to minimize the delays and improve the timely distribution of different messages. The designed technique minimizes the delay and improves the packet delivery ratio but the performance of packet loss was not evaluated. An efficient and reliable data dissemination protocol was introduced in [8] to handle the data distribution with acceptable delay. The designed protocol failed to improve the performance of the protocol in terms of other significant metrics such as the number of packet propagation, and intermediate hops between the source and the destination.

A Content-aware Stable Multimedia Communication (CSMC) algorithm was devised in [9] in order to increase the vehicle's communication stability and multimedia data communication of the cluster within a certain range. The designed algorithm minimizes the packet loss but optimal path identification remains unsolved to improve the data delivery. A quality of service-aware routing algorithm was proposed in [10] for obtaining the most reliable and connected path to transmit the packets. The designed algorithm improves the data delivery and minimizes the delay but the exact neighboring location identification was not performed.

A reliable low-latency multipath routing (RLLMR) method was introduced in [11] based on multipath link reliability for determining the reliable routes. The designed RLLMR method significantly minimizes the link failures but efficient stable link identification was not performed.

An enhanced intelligent hybrid routing protocol was introduced in [12] to discover the stable route path for data transmission between a source and a destination based on three significant factors of the vehicle such as speed, direction, and neighbor nodes. Connectivity modeling and investigation were proposed in [13] for IoV to improve the data transmission rate. Connectivity modeling results in increasing the reliability of data transmission. But it failed to identify the efficient neighbors with higher signal strength.

An intelligent forwarding-based stable and reliable data dissemination approach was presented in [14] for selecting the next forwarding node to transmit the data. However, the performance evaluation on other parameters such as packet loss rate and an end to end delay remained unaddressed.

Adaptive Beacon broadcast in the Opportunistic Routing (ABOR) technique [15] was introduced to improve the performance of data transmission. However, the designed ABOR technique failed to validate their impacts on the quality of services such as delivery rate and an end to end delay. In addition, an efficient mathematical model was not used to find the positional information of the nodes. An overlapping transmission system for IoVs was introduced in [16] to provide high reliability and less latency communication. But optimal path estimation was not performed.

A Link Reliability-Based Clustering Algorithm (LRCA) [17] was designed to offer efficient and reliable data communication by filtering the unstable neighbors. The designed algorithm failed to develop a routing strategy for minimizing the end-to-end delay. A hybrid relay node selection method was developed in [18] to use the best optimal features for message dissemination and minimize the communication delay. The performance of end to end the delay was minimized but efficient data dissemination was not achieved.

A Reliable Comprehensive Communication System for IoV (IoVCom) was developed in [19] to distribute the significant data between IoV components. Though the system minimizes the computational time, an efficient neighboring node selection was not performed.

A Multi-Hop Clustering approach was designed in [20] to enhance the transmission performance between Vehicleto-Internet. The designed approach reduces message delivery latency and improves the message delivery ratio. Routing Selecting Based on the Link Quality (RSBLQ) estimation method was designed in [21] to find stable routes with minimum average delay. The RSBLQ estimation method failed to use a better routing scheme for enhancing the link quality.

The core contribution of the paper is summarized as follows:

- A new TLIMWDST technique is proposed to find the reliable route path that considers the expected packet loss rate and improves data delivery through the connectivity of vehicular nodes.

- Initially, the signal strength of vehicle node is measured for finding the location of neighboring node. The trilateration process determines the exact coordinate of neighboring vehicles using the geometry of circles through the point of intersection.

- Secondly, the TLIMWDST technique uses the Maximum Weighted Directive Spanning Tree to construct the directed graph which consists of vertices and edges. The vertices are lines based on the weight assignment. The maximum weighted links are connected and form a route from source to destination. This link is established based on the delay variation of beacon message arrival and transmission. The link with lesser delay variation is selected to transmit the data. As a result, the higher packet delivery ratio is achieved with minimum end to end delay. 
- Finally, the performance of the proposed TLIMWDST technique is conducted under a simulated scenario based on different performance metrics.

The paper is organized into six different sections. Section 2 describes the proposed TLIMWDST technique with different phases. Then, extensive simulations settings are described in Section 3. Followed by, the performance evaluations under different metrics are presented in section 4. In Section 5, the related works are reviewed. Finally, section 6 provides the conclusion.

\section{TRILATERAL LOCATION IDENTIFIED MAXIMUM WEIGHTED DIRECTIVE SPANNING TREE FOR DATA DELIVERY IN IOV}

VANET is a type of wireless network which utilizes the vehicles as nodes for the data communication. The vehicles in IoV are communicated and data from the vehicles communicate with other vehicles through the internet. In this case, an optimal route path is required to achieve reliable communication. The novel TLIMWDST technique performs the data transmission among the multiple communities. In IoV, multiple communities are formed based on the vehicle moving along the same destination. Multiple communities are connected to multiple access points in the network.

In this section, a novel TLIMWDST technique is introduced for increasing the reliability of data transmission from source to destination vehicle by implementing the two processes, namely location identification and optimal path identification. The process of the TLIMWDST technique is illustrated in Fig. 1.

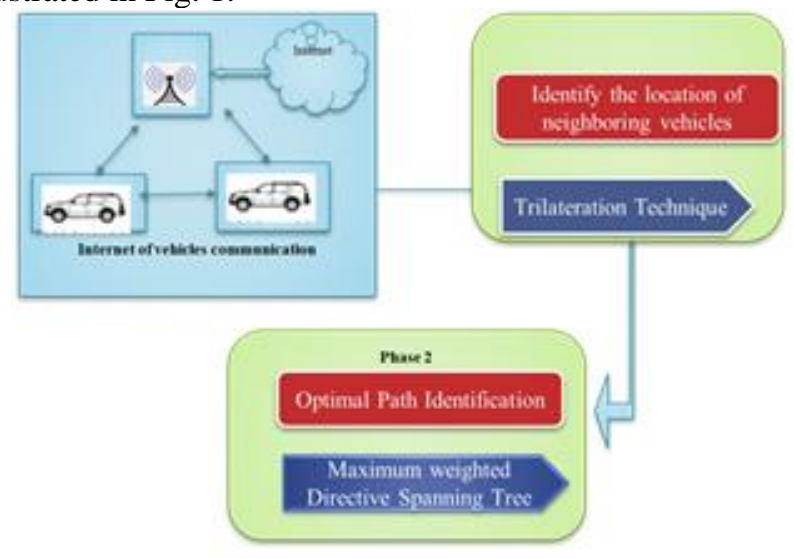

Figure 1. Basic communication structure of TLIMWDST technique

Fig. 1 illustrates the basic communication structure of the TLIMWDST technique where the network architecture comprises of internet, RSU and vehicles. The data communication is performed between the vehicles and vehicles to RSU. The vehicles $v_{1}, v_{2}, v_{3}, \ldots . v_{n}$ are deployed in a square area of ' $n * n$ '. Among the distributed vehicles, the source vehicle $(S V)$ is identified and it transmits the data packets $D p_{i}=D p_{1}, D p_{2}, D p_{3}, \ldots D p_{n}$ to the destination vehicle $(D V)$ through the intermediate nodes $N n_{1}, N n_{2}, N n_{3}, \ldots . N n_{n}$. The vehicles moving towards the same destination have to form different communities for data transmission. Before the data transmission, the source node finds the neighboring location by applying the trilateration technique. Then the optimal route path $\left(P_{t h}\right)$ is identified using Maximum Weighted Directive Spanning Tree for efficient data communication.

\section{A. LOCATION IDENTIFICATION USING TRILATERATION TECHNIQUE}

The first phase of the TLIMWDST technique is to identify the location of the neighboring vehicles using the trilateration technique. The trilateration technique is a mathematical model used for location identification with the help of geometry of circles. Initially, the source vehicle finds the nearest vehicle which have higher signal strength. The received-signal-strength of the vehicle node is mathematically calculated as given below,

$$
R_{S S}=10 * \log _{10}\left[\frac{P_{T}}{P_{R}}\right] .
$$

According to (1), $R_{S S}$ indicates received signal power of the vehicle node, $P_{T}$ represents measured power, $P_{R}$ denotes reference power. The received signal power of the node is measured in terms of decibels $(\mathrm{dB})$. Then the source vehicle finds the nearest vehicle location which has maximum signal strength. The neighboring node location is identified through the trilateration process as shown in Fig. 2.

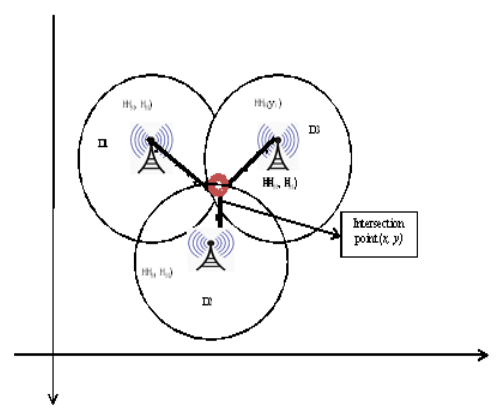

Figure 2. Trilateration process in Cartesian plan

Fig. 2 illustrates the trilateration process for finding the exact coordinates of the node in the Cartesian plane. Let us consider three different access points and the circle is drawn randomly around these three points. The circle around the access points is denoted as a signaling range.

As shown in above Fig. 2, three access points are located with three different distances represented by $D_{1}, D_{2}, D_{3}$ with coordinates $\left(x_{1}, y_{1}\right),\left(x_{2}, y_{2}\right),\left(x_{3}, y_{3}\right)$ and the intersection point is denoted as $(x, y)$. Therefore, the distance from the access point to the intersection is found using the distance formulas in the Cartesian plane as given below:

$$
\begin{aligned}
& D_{1}{ }^{2}=\left(x_{1}-x\right)^{2}+\left(y_{1}-y\right)^{2} . \\
& D_{2}{ }^{2}=\left(x_{2}-x\right)^{2}+\left(y_{2}-y\right)^{2} . \\
& D_{3}{ }^{2}=\left(x_{3}-x\right)^{2}+\left(y_{3}-y\right)^{2} .
\end{aligned}
$$

From (2) (3) (4), the point with an intersection of three 
circles provides an exact position $(x, y)$ of a node in a given Cartesian plane. By applying the trilateration process, the distance is initially calculated between the access point and intersection and then the location is identified by solving the above nonlinear equations to get the Point Of Intersection $(P O I)$ as a vehicle's position. In this way, the locations of all neighbor's vehicles are identified for further processing in the VANET. After selecting the neighboring node, the route path from the source to the destination is established for data transmission.

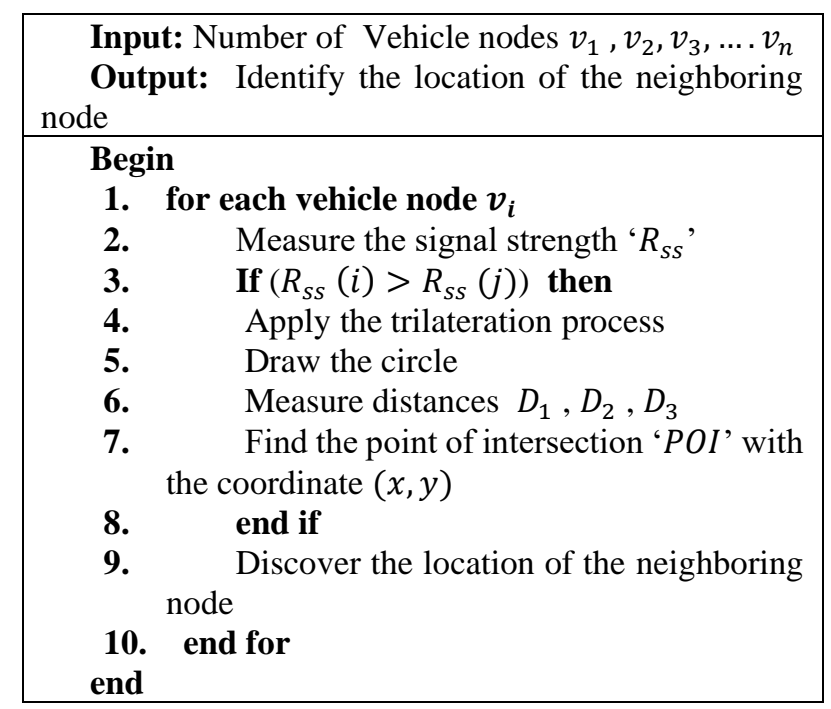

Algorithm 1 given above describes the location identification process to establish the route paths from the source vehicle to the destination vehicles. For each vehicle node in the network, the signal strength is measured for identifying the neighboring node. If the signal strength of the one vehicle $\left(R_{s s}(i)\right)$ is greater than the other $\left(R_{s s}(j)\right)$, then the trilateration process is applied to find the location of the neighboring node. Initially, the circles are drawn randomly around the access points and the point of intersection through the distance measure is found. The point at which the intersection of three coordinates is taken as the exact location coordinate of the vehicle node and is absolutely identified for optimal path identification.

\section{B. MAXIMUM WEIGHTED DIRECTIVE SPANNING TREE- BASED OPTIMAL PATH IDENTIFICATION}

The proposed technique finds an optimal route path among multiple paths using Maximum Weighted Directive Spanning Tree (MWDST). In graph theory, the spanning tree uses the directed graphical structure (G) which is mathematical formation used to link two points. In this perspective, a graph (G) is constructed with a set of vertices (v) (also called vehicle nodes) which are connected by edges (i.e., links between the nodes). In MWDST, the nodes are connected, where all the links are directed from one node to another using the right arrow. A directed graph is also called a directed network. Initially, the source vehicle sends a beacon message to find the next forwarding neighboring node.

$$
\mathrm{SV} \stackrel{\mathrm{b}_{\mathrm{msg}}}{\longrightarrow} \mathrm{Nn}_{\mathrm{i}} \text {. }
$$

In (5), SV denotes a source vehicle, $\mathrm{Nn}_{\mathrm{i}}$ denotes neighboring nodes, $b_{\mathrm{msg}}$ represents the beacon message. After receiving the beacon message, the neighboring node $\left(\mathrm{Nn}_{\mathrm{i}}\right)$ sends the beacon message to another neighboring node $\left(\mathrm{Nn}_{\mathrm{j}}\right)$ as given below:

$$
\mathrm{Nn}_{\mathrm{i}} \stackrel{\mathrm{b}_{\mathrm{msg}}}{\longrightarrow} \mathrm{Nn}_{\mathrm{j}} \text {. }
$$

Based on the beacon message distribution, the delay variation of the particular node is measured as given below:

$$
\Delta \mathrm{d}_{\mathrm{m}}=\mathrm{d}_{\mathrm{msg}}(\mathrm{A})-\mathrm{d}_{\mathrm{msg}}(\mathrm{S}) .
$$

In (7), $\Delta \mathrm{d}_{\mathrm{m}}$ denotes a delay variation, $\mathrm{d}_{\mathrm{msg}}(\mathrm{A})$ denotes a delay of beacon message arrival time of particular node(i), $\mathrm{d}_{\mathrm{msg}}(\mathrm{S})$ indicates the delay of the beacon message transmission from the one node (i) to another node (k). After computing the delay variation, the threshold is set to identify the next forwarded node to form a tree.

$$
y=\left\{\begin{array}{cc}
\Delta \mathrm{d}_{\mathrm{m}}<\beta & , \text { Select forwarding node } \\
\text { Otherwise, } & \text { find other node }
\end{array} .\right.
$$

In (8), y denotes output results, $\beta$ denotes a threshold, $\Delta \mathrm{d}_{\mathrm{m}}$ indicates a delay variation. The node with the delay variation less than the threshold is selected as the next forwarding node. Otherwise, the node is selected as a forwarding node. The maximum weight is given to the less delay variation than the other node. The maximum weighted link is chosen for the optimal path construction. The weight is an integer number which is assigned to each link between the nodes.

Fig. 3 illustrates the Maximum Weighted Directive Spanning Tree to find the optimal path from the source to the destination vehicle. The graph comprises the ' $n$ ' vertices and $n-1$ edges.

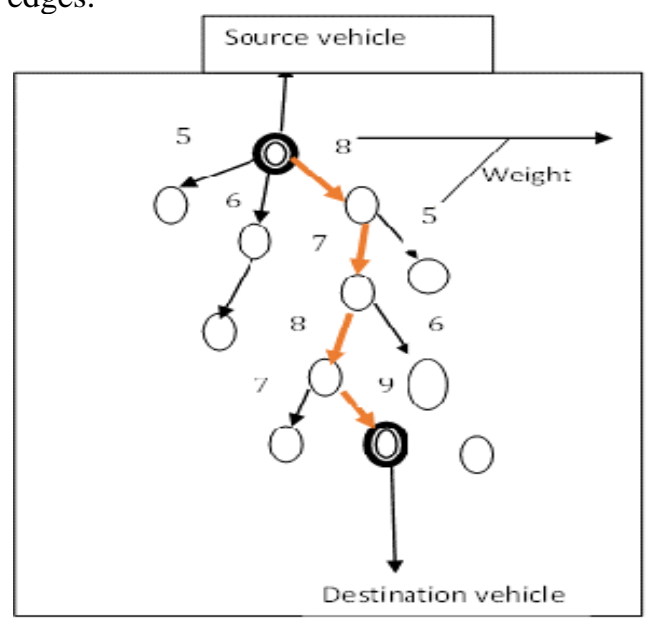

Figure 4. Maximum Weighted Directive Spanning Tree 
As shown in Fig. 3, the red color arrow mark indicates an optimal route path from the source to the destination. Then the source vehicle transmits data packets to a destination via selected route path. The algorithmic process of Maximum Weighted Directive Spanning Tree optimal path selection is described as follows:

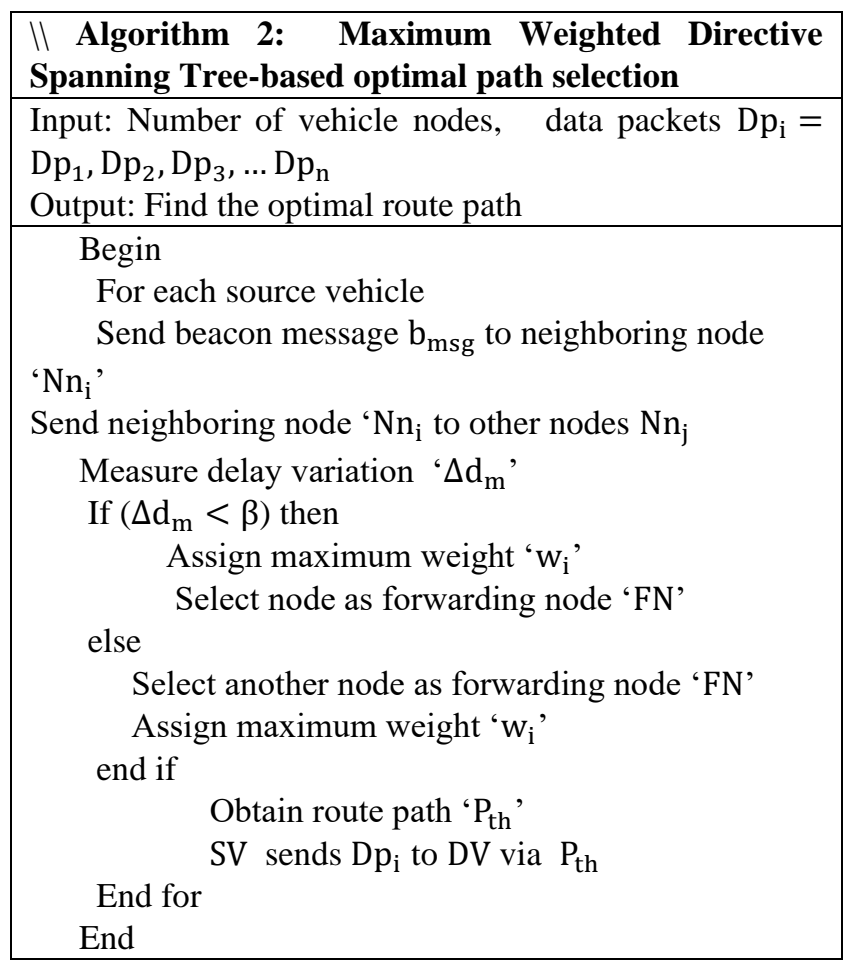

Algorithm 2 explains the step by step process of optimal route path selection for data transmission. Initially, the source vehicle sends a beacon message to the neighboring vehicle with a certain amount of delay. These delay variation is measured for identifying the next forwarded node. If the delay variation is less than the threshold, then the link is assigned higher weight and the node is selected as the next forwarded node. In this way, the nodes and links are identified until the destination is reached. The link having the minimum weight is not selected for data transmission. If all nodes have the same delay variation the neighboring node is selected using the Breadth first Search (BFS).The maximum weighted link between the nodes increases the data delivery and reduces the packet loss. Then the source node sends the data packet to destination along the route path which improves data delivery.

\section{SIMULATION SETTINGS}

In this section, the TLIMWDST technique and three existing methods, namely CIoVS [1], RPSPF [2], IDCIoV [3] are implemented in the NS2.34 network simulator. In the simulation scenario, 500 vehicle nodes are deployed in a square area of $A^{2}(1100 \mathrm{~m} * 1100 \mathrm{~m})$. For the simulation purposes, the random waypoint mobility model and DSR protocol are used for data transmission. In order to conduct the simulation, the total time is set as $300 \mathrm{sec}$. The various simulation parameter values are listed in Table 1.

Table 1. Simulation parameters

\begin{tabular}{|l|c|}
\hline $\begin{array}{l}\text { Simulation } \\
\text { Parameters }\end{array}$ & Values \\
\hline Network Simulator & NS2.34 \\
\hline Square area & $1100 \mathrm{~m} * 1100 \mathrm{~m}$ \\
\hline $\begin{array}{l}\text { Number of vehicle } \\
\text { nodes }\end{array}$ & $50,100,150,200,250,300,350,400,450$, \\
\hline $\begin{array}{l}\text { Number of data } \\
\text { packets }\end{array}$ & $30,60,90,120,150,180,210,240,270,30$ \\
\hline Mobility model & Random Waypoint model \\
\hline Speed of nodes & $0-20 \mathrm{~m} / \mathrm{s}$ \\
\hline Simulation time & $300 \mathrm{sec}$ \\
\hline Protocol & DSR \\
\hline Number of runs & 10 \\
\hline
\end{tabular}

\section{RESULTS AND DISCUSSION}

The performance of the TLIMWDST technique is evaluated with three existing methods, namely CIoVS [1], RPSPF [2], IDCIoV[3] using the performance metrics such as packet delivery ratio, packet loss rate, end to end delay and throughput. The simulation results are described with the help of a table and graphical representation.

The first performance metric is packet delivery ratio which is defined as the ratio of a number of (i.e., no. of) successfully received data packets to the total number of data packets sent. The packet delivery ratio is estimated using the following expression:

$$
\mathrm{PDR}=\left[\frac{\text { Number of data packets received }}{\text { Number of data packet sent }}\right] * 100,
$$

where PDR denotes a packet delivery ratio and it is measured in terms of percentage (\%). The performance analysis of the packet delivery ratio using the TLIMWDST technique and three existing methods, namely CIoVS [1], RPSPF [2], IDCIoV[3] are illustrated with the help of a graph.

The TLIMWDST technique outperforms well in terms of achieving the higher data delivery ratio than the other related approaches. This considerable enhancement of the TLIMWDST technique is achieved through the exact location identification and reliable path selection. This is proved by the statistical evaluation. The observed results of the TLIMWDST technique are compared to the existing methods. The average of ten results signifies that the TLIMWDST technique considerably increases the performance of the packet delivery ratio by $11 \%$ when compared to CIoVS [1] and 7\% when compared to RPSPF [2] and 3\% when compared to IDCIoV[3] respectively. The graphical illustration of the packet delivery ratio is shown in Fig. 4. 


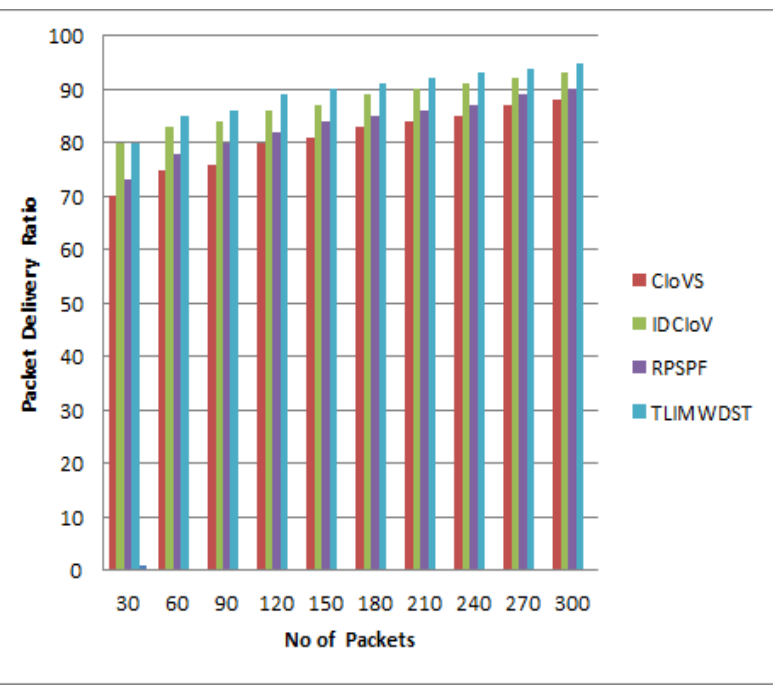

Figure 4. Impact of packet delivery ratio

Fig. 4 presents the impact of packet delivery ratio versus the number of data packets. An optimal forwarded node selection is used for effectively providing a higher packet delivery ratio as compared to CIoVS [1], RPSPF [2], IDCIoV[3].

The second performance metric is packet loss rate which is referred to a ratio of a number of data packets lost during the data transmission to the total number of data packets sent from the source vehicles. The mathematical formula for calculating the packet loss rate is expressed as follows:

$$
\text { PLR }=\left[\frac{\text { Number of data packets lost }}{\text { Number of data packet sent }}\right] * 100,
$$

where PLR indicates a packet loss rate measured in terms of percentage (\%). The performance analyses of the packet loss rate using three path selection methods are illustrated in Fig. 5.

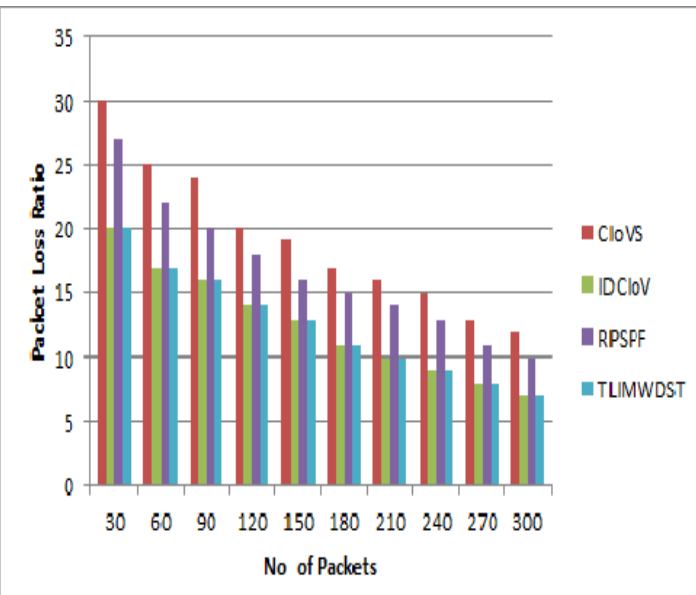

Figure 5. Impact of packet loss rate

Fig. 5 shows the simulation outcomes of packet loss rate versus the number of data packets. The graphical plot illustrates that our TLIMWDST technique minimizes the packet loss rate for forwarding the data packets through the stable links. The stable links are obtained through the maximum weighted directive spanning tree. The tree is constructed based on the maximum weighted directed link between the two nodes.

Due to the selection of a maximum weighted link, the stable route path from the source to the destination is established. Finally, this route path is chosen as an optimal path for efficient data transmission. This helps to show that the TLIMWDST technique contains a tree-based path selection mechanism resulting in it achieves the considerable improvement over other approaches. It enhances the performance of the data delivery by providing the paths with better connectivity.

The third performance metric is an end to end delay which is referred to as the time difference between the data packet arrival and sending from the source to the destination. The overall end to end delay is computed using the given formula

$$
\mathrm{EED}=\left[\mathrm{Dp}_{\mathrm{Arr}}\right]-\left[\mathrm{Dp}_{\mathrm{s}}\right],
$$

where EED indicates an end to end delay, $\mathrm{Dp}_{\mathrm{Arr}}$ symbolizes the data packet arrival time, $\mathrm{Dp}_{\mathrm{s}}$ indicates the data packet sending time. The measurement of delay is performed in terms of milliseconds (ms). Less is the end to end delay, the technique is said to be more efficient.

It shows that an increase in the number of packets has a considerable impact on the performance of the TLIMWDST technique as compared to the considered existing routing techniques. This is because of its novel TLIMWDST technique which incorporates the location identification process and reliable forwarding which provides better connectivity for routing in a better way as compared to earlier techniques The results are illustrated in Fig. 6.

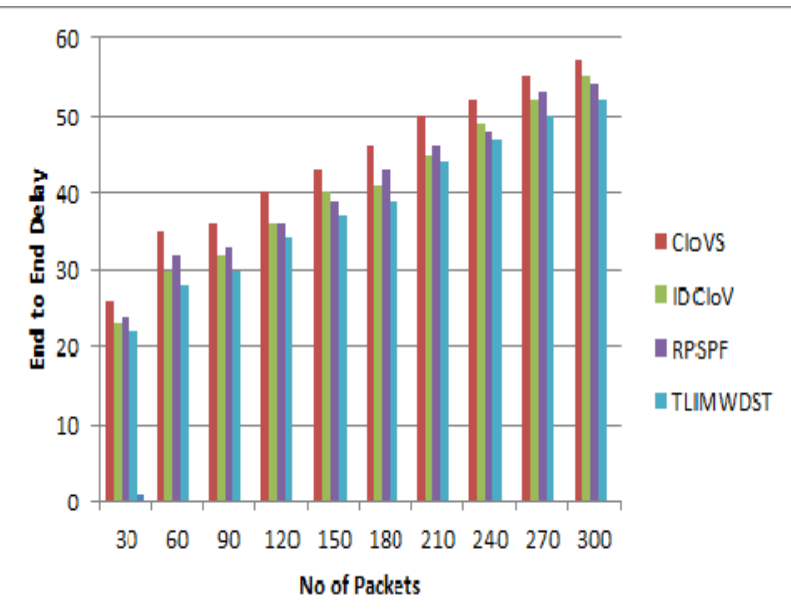

Figure 6. Impact of end to end delay

Fig. 6 exhibits the simulation results of the end to end delay using different count of data packets. As shown in Fig. 6 , the end to end delay for the proposed TLIMWDST 
technique is better as compared to all the other considered existing techniques and therefore, it means that less is end to end delay. Based on the figure, it is inferred that the TLIMWDST technique reduces the delay. The higher signal strength node selection of the TLIMWDST technique is to deliver the data packets continuously with less delay.

Finally, the fourth performance metrics is a throughput which is defined as the amount of data delivered at the destination in a given period of time.

$$
\text { Throughput }=\frac{\text { Amount of } D_{\text {preceived }(\text { bits })}}{\text { time }(\text { second })}
$$

where, ' $D_{\mathrm{p}}$ ' denotes the data packets. Throughput is computed in bits per second (bps).

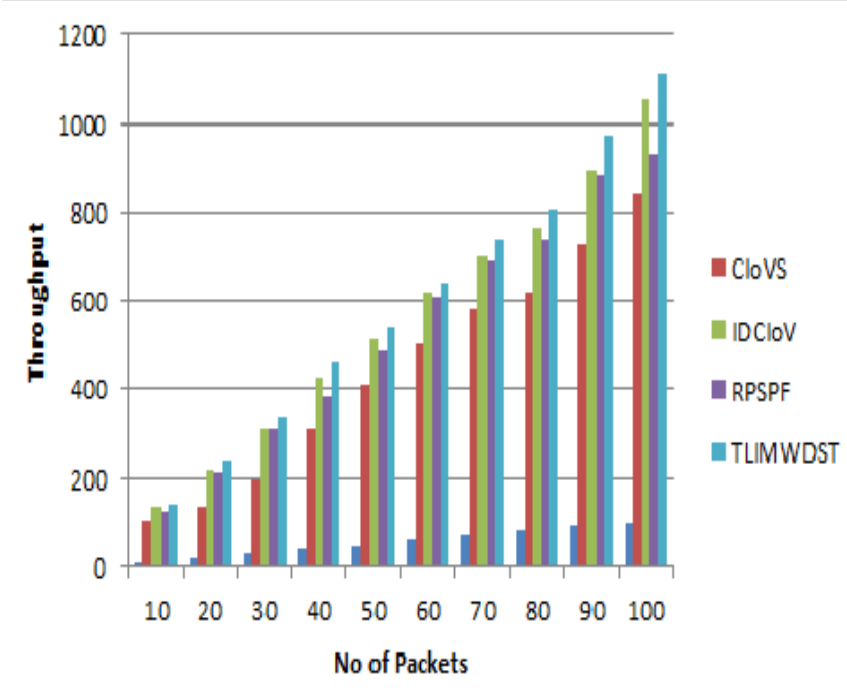

Figure 7. Impact of throughput

Fig. 7 depicts the simulation outcome of throughput based on the number of data packet size. The number of data packet size is taken as input and it varied in the ranges of $10 \mathrm{~KB}$ to $100 \mathrm{~KB}$. According to the above figure, the throughput of the proposed TLIMWDST technique is much higher than that of the other methods. This is because of the application of MWDST for determining the optimal route path between the source and destination. With this, the amount of data packet received is considerably increased than with the use of the other methods.

\section{CONCLUSION}

In this paper, a novel technique over IoV called TLIMWDST is developed for improving data transmission performances. IoV allows the vehicles to connect to the Internet via a unique infrastructure called a Road Side Unit hence that each vehicle receives and distributes the essential information about its neighbor's location to perform the data transmission. The above said objectives of the IoV communication are achieved using a trilateration location identification based maximum weighted directive spanning tree. In the TLIMWDST technique, neighboring location identification is carried out by the trilateration mathematical procedure which is derived based on the distance equations and obtains the exact intersection coordinates of the node. Followed by, the graph is traversed using a maximum weighted directive spanning tree based on a delay variation of the link between the nodes and the maximum weights. As a result, the optimal route from the source to the destination is identified. Our simulation uses the NS-2 network simulator to analyze the performance of the TLIMWDST technique.

\section{References}

[1] T. Qiu, X. Liu, K. Li, Q. Hu, A. K. Sangaiah, N. Chen, "Communityaware data propagation with small world feature for the internet of vehicles," IEEE Communications Magazine, vol. 56, issue 1, pp. 8691, 2018. https://doi.org/10.1109/MCOM.2018.1700511.

[2] I. A. Abbasi, A. S. Khan and S. Ali, "A reliable path selection and packet forwarding routing protocol for vehicular ad hoc networks," EURASIP Journal on Wireless Communications and Networking, Springer, vol. 2018, pp. 1-19, 2018. https://doi.org/10.1186/s13638018-1233-z.

[3] S. Suguna Devi and A. Bhuvaneswari, "Identical destination based community on internet of vehicles (IDCIoV) for optimal path identification," Proceedings of the International Conference on Sustainable Communication Networks and Application, 2019, pp. 368-378. https://doi.org/10.1007/978-3-030-34515-0_40.

[4] H. H. R. Sherazi, Z. A. Khan, R. Iqbal, S. Rizwan, M. A. Imran, and $\mathrm{K}$. Awan, "A heterogeneous IoV architecture for data forwarding in vehicle to infrastructure communication," Mobile Information Systems, Hindawi, vol. 2019, pp. 1-12, 2019. https://doi.org/10.1155/2019/3101276.

[5] W. Zhu, D. Gao, C. H. Foh, H. Zhang, H.-C. Chao, "Reliable emergency message dissemination protocol for urban internet of vehicles,"IET Communications, vol. 11, issue 8, pp. 1275-1281, 2017. https://doi.org/10.1049/iet-com.2016.0661.

[6] W. Zhu, D. Gao, W. Zhao, H. Zhang \& H.-P. Chiang, "SDN-enabled hybrid emergency message transmission architecture in internet-ofvehicles," Enterprise Information Systems, vol. 12, issue 4, pp. 1-21, 2018. https://doi.org/10.1080/17517575.2017.1304578.

[7] M. Ali, A. W. Malik, A. U. Rahman, S. Iqbal, and M. M. Hamayun, "Position-based emergency message dissemination for the Internet of Vehicles," International Journal of Distributed Sensor Networks, vol. 15, issue 7, pp. $1-12, \quad 2019$ https://doi.org/10.1177/1550147719861585.

[8] S. Latif, S. Mahfooz, N. Ahmad, B. Jan, H. Farman, M. Khan, and K. Han, "Industrial internet of things based efficient and reliable data dissemination solution for vehicular ad hoc networks," Wireless Communications and Mobile Computing, Hindawi, vol. 2018, pp. 116, 2018. https://doi.org/10.1155/2018/1857202.

[9] K. Lin, F. Xia, G. Fortino, "Data-driven clustering for multimedia communication in Internet of vehicles," Future Generation Computer Systems, Elsevier, vol. 94, pp. 610-619, 2019. https://doi.org/10.1016/j.future.2018.12.045.

[10] K. Z. Ghafoor, L. Kong, D. B. Rawat, E. Hosseini, A. S. Sadiq, "Quality of service aware routing protocol in software-defined internet of vehicles," IEEE Internet of Things Journal, vol. 6, issue 2, pp. 2817-2828, 2019. https://doi.org/10.1109/JIOT.2018.2875482.

[11] F. Abbas, P. Fan and Z. Khan, "A novel reliable low-latency multipath routing scheme for vehicular ad hoc networks," EURASIP Journal on Wireless Communications and Networking, Springer, volume 2018 , pp. 1-19, 2018. https://doi.org/10.1186/s13638-018-1292-1.

[12] S. R. Yahiabadi, B. Barekatain \& K. Raahemifar, "TIHOO: An enhanced hybrid routing protocol in vehicular ad-hoc networks," EURASIP Journal on Wireless Communications and Networking, $\begin{array}{lllll}\text { Springer, } & \text { volume } & 2019, & \text { pp. }\end{array}$ 
https://doi.org/10.1186/s13638-019-1503-4.

[13] J. Cheng, H. Mi, Z. Huang, S. Gao, D. Zang, C. Liu, "Connectivity modeling and analysis for internet of vehicles in urban road scene," IEEE Access, vol. 6, pp. 2692-2702, 2017. https://doi.org/10.1109/ACCESS.2017.2784845.

[14] M. Chahal and S. Harit, "A stable and reliable data dissemination scheme based on intelligent forwarding in VANETs," International Journal of Communication System, Wiley, vol. 32, issue 3, pp. 1-19, 2019. https://doi.org/10.1002/dac.3869.

[15] M. Naderi, F. Zargari, M. Ghanbari, "Adaptive beacon broadcast in opportunistic routing for VANETs," Ad Hoc Networks, Elsevier, vol. 86, pp. 119-130, 2019. https://doi.org/10.1016/j.adhoc.2018.11.011.

[16] D. Zhang, L. Zheng, Q. Chen, B. Wei, X. Ma, "A power allocationbased overlapping transmission scheme in internet of vehicles," IEEE Internet of Things Journal, vol. 6, issue 1, pp. 50-59, 2019. https://doi.org/10.1109/JIOT.2018.2873480.

[17] X. Ji, H. Yu, G. Fan, H. Sun, and L. Chen, "Efficient and reliable cluster-based data transmission for vehicular ad hoc networks," Mobile Information Systems, Hindawi, vol. 2018, pp. 1-15, 2018. https://doi.org/10.1155/2018/9826782.

[18] O. Rehman, M. Ould-Khaoua, "A hybrid relay node selection scheme for message dissemination in VANETs," Future Generation Computer Systems, Elsevier, vol. 93, pp. 1-17, 2019. https://doi.org/10.1016/j.future.2018.10.042.

[19] T. Limbasiya and D. Das, "IoVCom: Reliable comprehensive communication system for internet of vehicles," IEEE Transactions on Dependable and Secure Computing, pp. 1-15, 2019. https://doi.org/10.1109/TDSC.2019.2963191.

[20] O. Senouci, Z. Aliouat, S. Harous, "MCA-V2I: A multi-hop clustering approach over vehicle-to-internet communication for improving VANETs performances," Future Generation Computer Systems, Elsevier, vol. 96, pp. 309-323, 2019. https://doi.org/10.1016/j.future.2019.02.024.

[21] L. Rui, H. Guo, R. Shi, H. Huang, and X. Qiu, "ICN routing selecting scheme based on link quality for the urban vehicles' communication," EURASIP Journal on Wireless Communications and Networking, Springer, vol. 2017, pp. 1-21, 2017. https://doi.org/10.1186/s13638-

\section{7-0991-3}

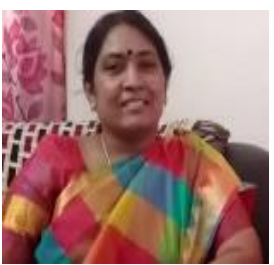

MS. S. SUGUNA DEVI pursued Master of Computer Applications from Bharathidasan University in the year 1999 and has 15 years of teaching experience. She is currently pursuing Ph.D. and working as Assistant Professor in the Department of Computer Science, Cauvery College for Women Trichy, Tamil Nadu, India. She is a life member of ISSE. Her main research work focuses on Internet of Vehicles.

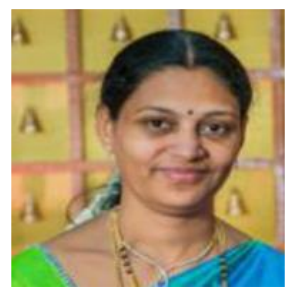

Dr. A. BHUVANESWARI completed her Masters Degree in Computer Science and Master of Philosophy in Computer Science in the year 2002 and 2005 respectively. She has also completed her Doctorate in Computer Science in the year 2015. She has around 18 years of Academic experience and eleven

years of Research experience in the field of Computer Science. Currently she is working at Cauvery College for Women (under the affiliation of Bharathidasan University), Trichy, Tamil Nadu, India. She has published several papers in International Journals and Conferences related to Computer Science. Her area of interest is Mobile Communication and Internet of Things. 